\title{
Decontamination of Surfaces Exposed to Single Wall Carbon Nanohorns
}

\author{
Z. Karimi ${ }^{1}$, P. Su ${ }^{2}$, R. Oftadeh ${ }^{1}$, H. Ebrahimi ${ }^{1}$, R. Ghosh ${ }^{1}$, and A. Vaziri ${ }^{1, *}$ \\ ${ }^{1}$ Department of Mechanical and Industrial Engineering, Northeastern University, Boston MA, \\ 02115 USA \\ ${ }^{2}$ FM Global, Norwood MA, 02062 USA \\ * Correspondence should be addressed to: vaziri@coe.neu.edu
}

\begin{abstract}
The effect of different surfactants on the removal efficiency of wiping surfaces contaminated with single walled carbon nanohorns (SWCNHs) was studied. To this end, sodium dodecylbenzenesulfonate (SDBS) and sodium dodecyl sulfate (SDS) surfactants were used, and their removal efficiencies with water only and with cleaning with a dry wipe were compared. The surfactant concentrations and wipe pressure during the wiping process were varied, and significant effects on removal efficiency were found. In addition, the results were compared with those obtained with single-walled carbon nanotubes (SWCNTs) and multi-walled CNTs (MWCNTs) and the differences among these nanostructures were reported. The results suggest that SDS and SDBS are good candidates for removal of SWCNHs deposited on silicon wafers with SDS removal efficiencies capable of exceeding 90\%. In addition, the results show that there is an optimum wiping pressure and surfactant concentration with the highest removal efficiency. A direct relationship was also found between wipe saturation and removal efficiency of SWCNHs deposited on silicon substrates. The differences between individual nano structures were perceptible in spite of following similar broad trends; for instance, SWCNH contaminated surfaces in general proved more difficult to clean than surfaces contaminated with the other nanostructures.
\end{abstract}




\section{Introduction}

Carbon based nanostructures, including single walled carbon nanohorns (SWCNHs), single walled carbon nanotubes (SWCNTs), and multi walled carbon nanotubes (MWCNTs), have garnered tremendous interest in recent times due to their non-traditional properties. For instance, carbon nanotubes (CNTs) exhibit exceptional mechanical properties, unique electrical conductivity and high field emission characteristics [1-8]. SWCNHs, which were discovered a few years after CNTs, are about $40-50 \mathrm{~nm}$ in tubule length and about $2-3 \mathrm{~nm}$ in diameter. They are derived from SWCNTs and terminated by a five-pentagon conical cap with a cone opening angle of $\sim 19^{\circ}[9]$. In contrast to CNTs, thousands of SWCNHs associate with each other to form 'dahlia-like' and 'bud-like' structured aggregates which have an average diameter of about 80$100 \mathrm{~nm}$. Figure 1 shows the transmission electron microscope (TEM) imaging of aggregation of these three types of carbon nanostructures: SWCNHs, SWCNTs and MWCNTs. Similar to CNTs, SWCNHs also hold tremendous possibilities for a wide range of potential applications such as energy management systems, medical applications such as drug delivery, gas absorption and synthesis of compound materials [10-12]. However, in contrast to the promise of high performance material behavior, these nano structures have also raised concerns regarding biological toxicity particularly due to their high surface to volume ratio, high reactivity and needle-like structures [13-18]. Thus, better control of nanostructure exposure and decontamination are an important goal for safe and convenient handling of these materials. Unfortunately, this task has proved to be challenging. For instance, removal of CNTs in case of accidental release in the work space can be difficult due to the strong adhesion between the CNTs and the substrate. This adhesion is caused by the high surface contact area and an external force is required to separate the CNTs from the substrate [19-21]. To remedy this undesired 
outcome, surfactants are widely used to disperse CNTs since they weaken the strong bond between particles using their inherent polarity. They also prevent the particles from re-adhesion, which is highly desirable for removal of CNTs from contaminated surfaces [22].

In this context, the ability of sodium dodecylbenzenesulfonate (SDBS) and sodium dodecylsulfate (SDS) surfactants to remove MWCNTs from rough surfaces was examined earlier [23]. Recently, we examined the removal of MWCNTs from silicon wafers using various surfactants: SDS, SDBS, gum arabic and calcium carbonate $\left(\mathrm{CaCO}_{3}\right)$ [23]. The study showed the superior ability of surfactants to remove CNTs deposited on silicon wafers compared to cleaning with a dry wipe or cleaning with water.

In the current study, we determined the removal efficiency of SWCNHs from SWCNHcontaminated silicon wafers using pre-saturated wipes with different saturation percentages and different surfactant concentrations. The results derived for SDBS and SDS surfactants were compared with water and cleaning with a dry wipe. We also compared the behavior of SWCNH with two other common nanostructures, SWCNT and MWCNT. Finally, the effects of different surfactant concentration, saturation and pressure on cleaning wipes of two different types on the removal efficiency were examined.

\section{Materials and Methods}

The MWCNTs and SWCNTs used for this study were combustion chemical vapor deposition (CCVD) grown and dispersed in polyvinylpyrrolidone (PVP) surfactant, whereas SWCNHs were produced using a continuous laser ablation method [23] and dispersed in ethanol. The average length of SWCNTs, MWCNTs and SWCNHs used in this study were $200 \mathrm{~nm}, 250$ $\mathrm{nm}$, and $45 \mathrm{~nm}$ and average diameters were $1.2 \mathrm{~nm}, 15 \mathrm{~nm}$, and $3 \mathrm{~nm}$, respectively. In the 
experiments, the nanostructures (CNTs and SWCNHs) were spin-coated on the wafers through a pristine liquid solution using a Laurell Spinner at $500 \mathrm{rpm}$ for $60 \mathrm{sec}$ and then heated to $105^{\circ} \mathrm{C}$ (glass transition temperature of PVP) for $90 \mathrm{sec}$. The wafers' diameter was $3 \mathrm{in}$. and the spin coating was performed for $1 \mathrm{~min}$ at $3000 \mathrm{rpm}$. A total number of 30 images with equal magnification and resolution were taken from different spots on each wafer surface using scanning electron microscope (SEM) imaging. The nanostructure surface aerial concentration for each image was then determined by an image processing code using MATLAB®. Average nanostructure aerial density from 30 different images of each wafer was obtained and used in the analysis.

For the first set of experiments, three different cleaning media of SDS (4\% technical grade in distilled water), SDBS (1.5\% technical grade in distilled water), and pure distilled water were used. First, the cleaning medium was sprayed on the surface of the nanostructurecontaminated silicon wafer. Next, after 2 minutes, the wafer was wiped once across the surface using cleanroom wipes (non-woven polyester/cellulose) which had been soaked in the cleaning medium (i.e., 100\% saturation) for added efficiency. The soaked wipes were mounted on a special device which was designed to control the applied pressure on the silicon wafer (see Figure 2). For this set of experiments, the wiping pressure and duration were $2 \mathrm{kPa}$ and $5 \mathrm{sec}$, respectively. The wafers were dried using nitrogen gas after the cleaning. After cleaning, the wafers were imaged using SEM and the average final aerial density for each wafer was obtained by post processing the images as explained earlier. The removal efficiency was obtained from observing the difference between the initial and the final average nanostructure aerial densities [24]. 
In the second set of experiments, SDS (1\% and 4\%) was chosen as the cleaning medium and the same decontamination process was performed on MWCNTs and SWCNHs deposited on the silicon wafers. At this stage, two different types of wipes (i.e. Polyester/Nylon Microdenier and EcoClean ${ }^{\circledR} 60$ - non-woven cotton) and three different saturation percentages (i.e. $45 \%$, $65 \%$ and $100 \%$ ) were used to explore the effect of wet wipe type and saturation on removal efficiency. Polyester/Nylon Microdenier is composed of polyester and nylon knitted microfiber microdenier, and in general shows high level of cleaning efficiency and absorption resistance. EcoClean $60 \AA$ is a nonwoven material, which in general provides low ionic trace and low fiber release. These two wipes have shown slightly better removal efficiency compared to cleanroom wipes (non-woven polyester/cellulose).

For the final set of experiments, SDS with $4 \%$ concentration was chosen to examine the effect of consecutive wipes and wipe pressure on removal efficiency of SWCNHs deposited on silicon wafers. In this set of experiments, SDS $4 \%$ was sprayed on the silicon wafer surfaces at first. After 2 minutes, cleanroom wipes (non-woven polyester/cellulose) which had been soaked in the SDS (i.e., 100\% saturation) were used for cleaning. The pressure was controlled by adding/removing weights to the high end cantilever of the designed pressure device (Figure 2).

\section{Results and Discussion}

The ability of different surfactants to remove the nanostructures from surfaces was quantified by the removal efficiency parameter, defined as the difference between the initial and final aerial density of the nanostructures divided by the initial aerial density. As seen in Figure 3A, the two surfactants used in the experiments, SDS and SDBS, tremendously improved the removal efficiency of SWCNHs (87\% for SDS and 77.5\% for SDBS) compared with pure water 
(43\%) or dry wiping (3.7\%). This trend is reflected across all nanostructures including the CNTs and can be attributed to the role of surfactant micelles in suspending the nanostructures in aqueous media as well as increasing the wettability of the surface by decreasing the water surface tension. In comparison to SWCNHs, the removal efficiency of SWCNTs and MWCNTs were consistently higher. This result can be attributed to the binding energy of the different nanostructures since the better cleaning of MWCNTs in comparison to SWCNTs is correlated to the larger value of the binding energy density holding the nanotube aggregates together for MWCNTs compared with SWCNTs [25]. Figure 3B-D shows the sample SEM images of SWCNH, SWCNTs and MWCNTs coated wafer surfaces before and after cleaning using different cleaning media.

Next, we studied the effect of wipes on the cleaning efficiency of SWCNHs and compared that with MWCNTs. To this end, Figure 4 shows the removal efficiency of SWCNHs and MWCNTs using SDS surfactant for two different types of wipes (i.e. Polyester/Nylon Microdenier and EcoClean 60 - non-woven cotton) and three different saturation percentages (i.e. $45 \%, 65 \%$ and $100 \%$ ). These two types of wipes showed the highest removal efficiency among four other types of wipes in a preliminary study. The figure shows that removal efficiency of SWCNHs is consistently improved with increase in wipe saturation from $45 \%$ to $100 \%$. However, the Polyester/Nylon Microdenier wipes showed marginally better cleaning performance compared with the EcoClean 60 - non-woven cotton for the same degree of saturation. Comparing Figure 4A with Figure 4B, it is clear that these broad trends hold for MWCNTs as well. Interestingly, the plots also show that, for a given wipe type and saturation, increasing SDS concentration from $1 \%$ to $4 \%$ results in a decrease of the removal efficiency for both nanomaterials. This finding is in agreement with previous results reported in the literature 
[26]. This apparent anomalous behavior can be explained by first noting that, when the surfactant concentration increases, the size of the surfactant micelles also undergoes an increase due to polar interactions. However, if the surfactant concentration surpasses an optimum value, the surface area of micelles becomes so large that, instead of interacting with the nanostructures, they begin to interact with each other thereby decreasing the removal efficiency [27]. Furthermore, if the outer layer of surfactants orients in such a way that the hydrophobic tails extend into the solution, the surfactant's surface energy decreases and dispersion of CNTs also decreases. This phenomenon is known as Flocculation [28]. In this context, Sun et al. [29] obtained the optimum concentration of some surfactants for suspending CNTs as $1 \%$ and Rastogi et al. found it to be equal to $1.3 \%$ for SDS. [26]. In addition to these common trends, contrasting Figure 4A and 4B also indicates that the better removal efficiency of MWCNT over SWCNH observed earlier is also reproduced in the current set of experiments for corresponding wipe saturation and wipe type across all surfactant concentrations except for the single case of $4 \%$ SDS and 45\% saturated Polyester/Nylon Microdenier wipe. Figure 5 shows the effect of different wiping pressure and the number of consecutive wipes on the removal efficiency of SWCNHs. As can be seen from the figure, at each specific pressure, as the number of consecutive wipes increases, the removal efficiency of SWCNHs from the substrate also increases. However, we find a significant nonlinear convergence effect with increase in pressure; beyond $7 \mathrm{kPa}$ pressure, removal efficiencies become very high even with a single wipe, thus exhibiting a plateau type effect for subsequent wiping.

\section{Summary}

In summary, the removal efficiency of SWCNHs deposited on silicon wafers for different types of surfactants and types of wipe and their saturation percentages were examined and 
contrasted with more common CNTs in this work. The results clearly demonstrate that surfactants (SDS and SDBS) can significantly enhance cleaning of nanostructures from a silicon substrate compared to water or cleaning with a dry wipe. Further improvements could also be fostered by an adroit control of wipe saturation percentages, surfactant concentration and wipe application pressure. In addition, surfactants are economical, relatively easy to use and commercially available. These factors make surfactants a good candidate for the removal of SWCNHs deposited on surfaces that are often caused by unwanted spillage, thus preventing their further accidental exposure in the work space or environment. Finally, our study also demonstrates that, even within the broad trends, perceptible differences remain between the responses of individual nanostructures. Therefore, further studies need to be conducted to determine the effectiveness of removal of CNTs and SWNHs obtained from various production methods or with different chemical modifications.

\section{Acknowledgments}

The work was supported by the National Science Foundation CMMI Grant- 1149750 and FM Global. The authors would like to thank Berkshire Company for providing the wipe samples for this paper. 
(A)
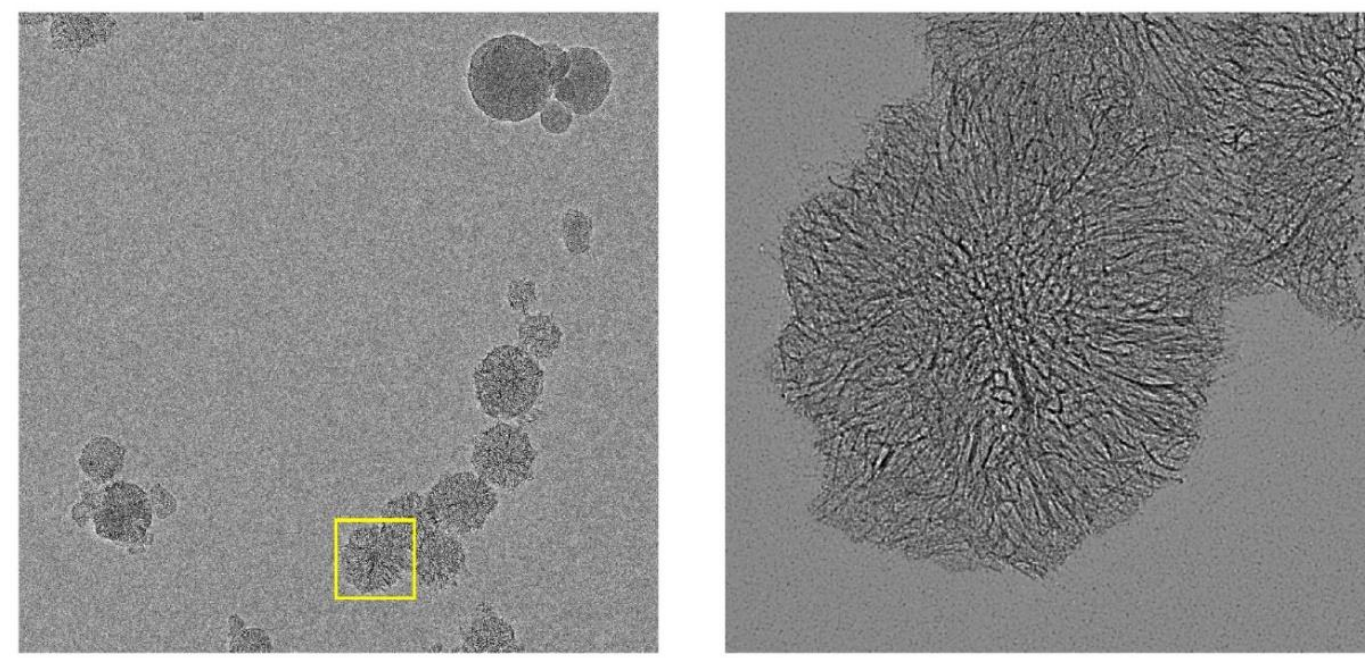

(B)
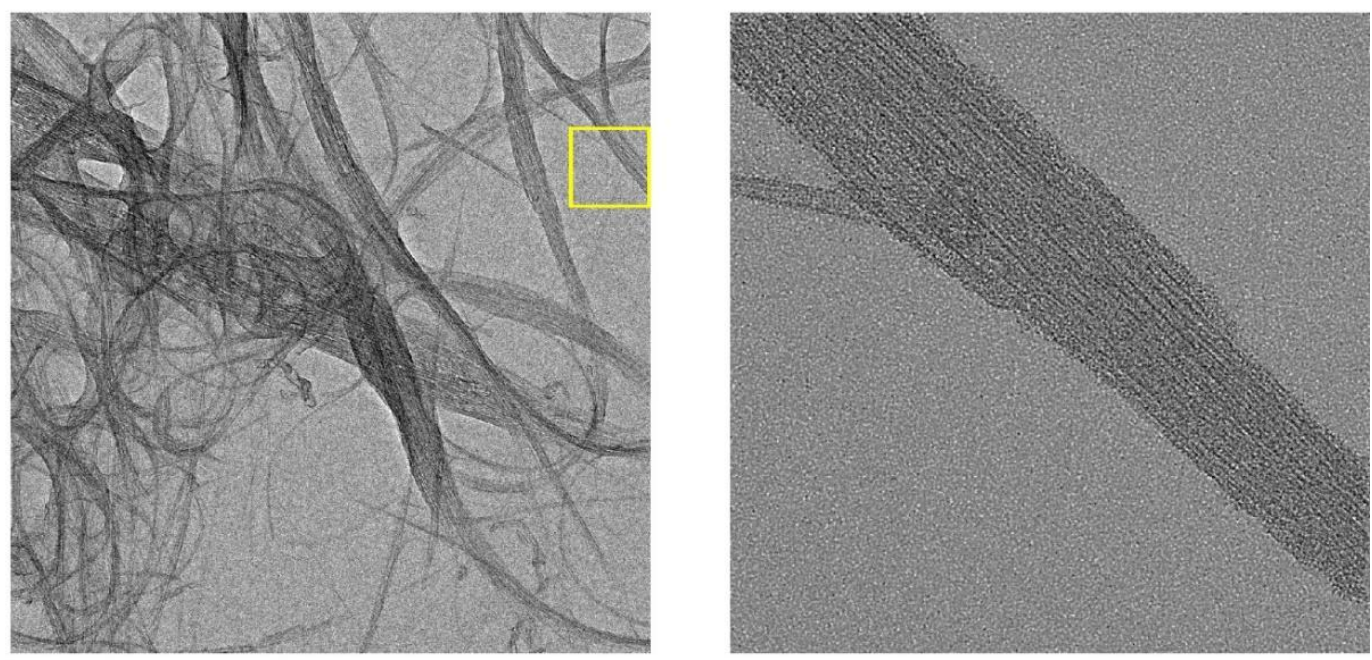

(C)
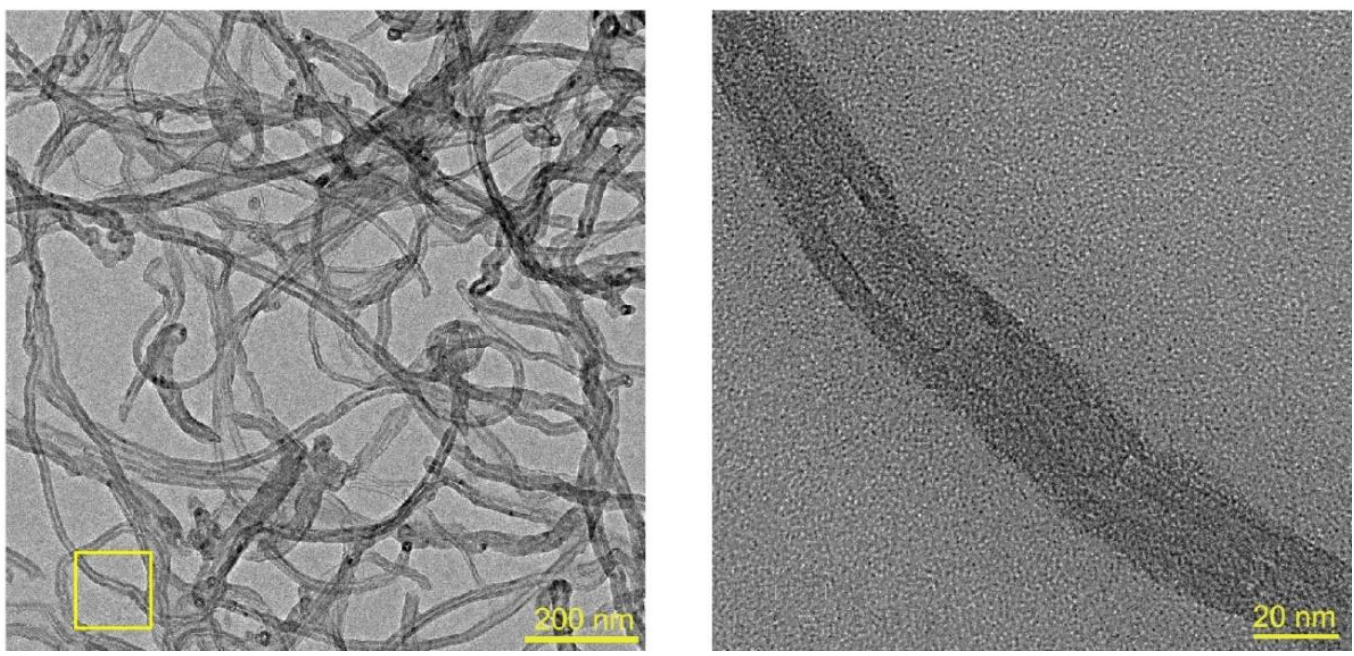

Fig. 1 (A) TEM imaging of aggregation of carbon nanohorns (diameter approximately $100 \mathrm{~nm}$ ) (B) Aggregation of single walled carbon nanotubes (C) Aggregation of multi walled carbon nanotubes. 

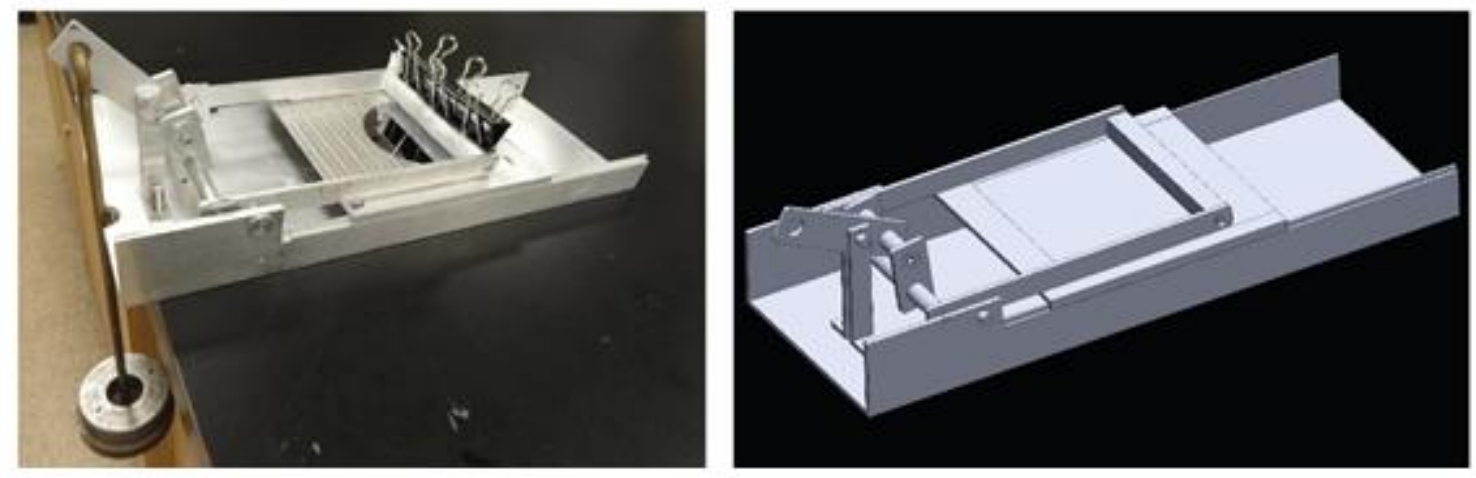

Fig. 2. Device designed to control the platform pressure which is imposed on the silicon wafer. A photograph of the device is at left, and a $3 \mathrm{D}$ computer aided representation of the device is at right. 
(A)

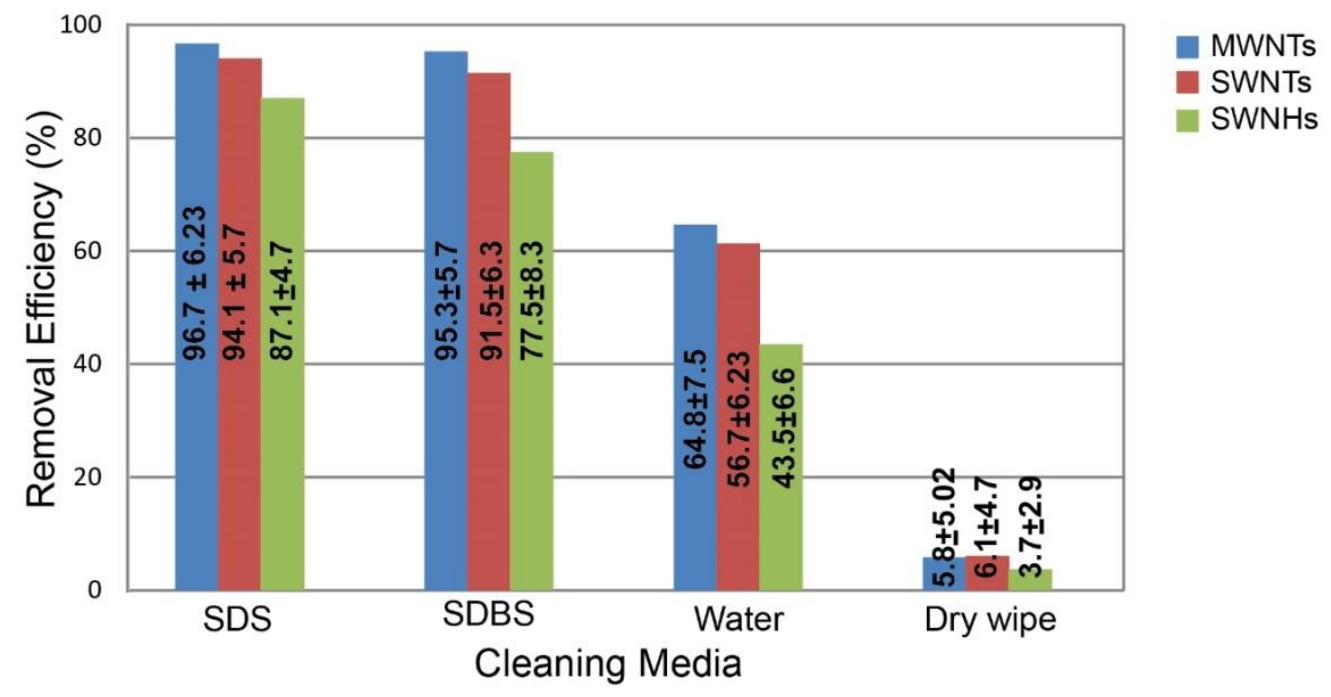

(B)
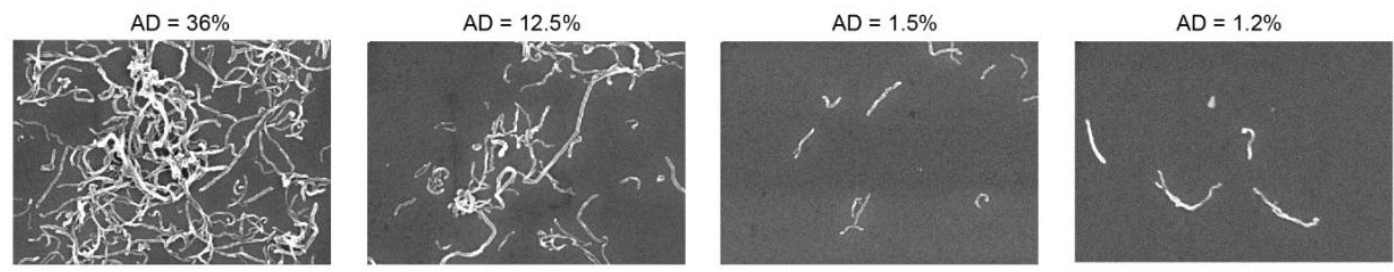

(C)

PD
$\mathrm{AD}=38 \%$

Post cleaning (pure water) $A D=14.7 \%$

Post cleaning (SDBS) $A D=3.2 \%$

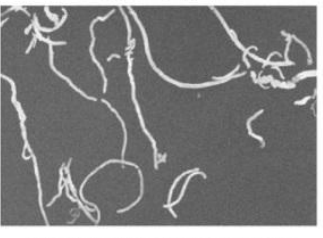

Post cleaning (pure water) $A D=27.3 \%$

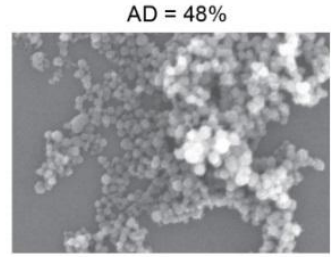

Pre-cleaning

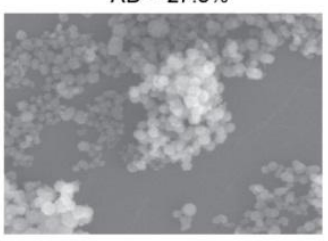

Post cleaning (pure water)

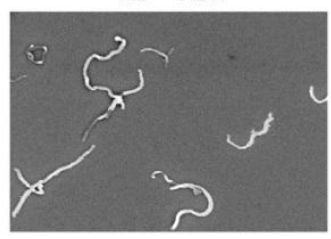

Post cleaning (SDBS)

$A D=10.8 \%$

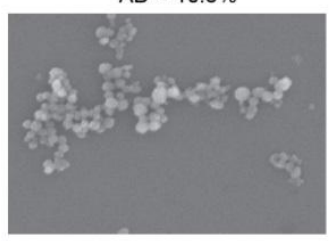

Post cleaning (SDBS)
Post cleaning (SDS) $A D=2.1 \%$

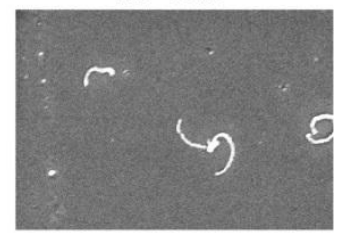

Post cleaning (SDS)

$A D=7.9 \%$

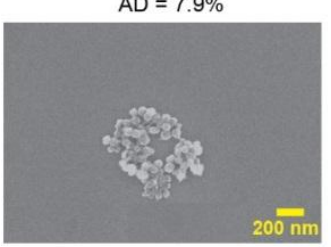

Post cleaning (SDS)

Fig. 3 (A) Removal efficiency of SDS, SDBS, water, and dry wiping on multi-walled carbon nanotubes (MWCNT), single-walled carbon nanotubes (SWCNT) and single-walled carbon nanohorns (SWCNH) (B) Sample SEM image of the surface of MWCNTs deposited silicon wafers before cleaning (Aerial Density $(\mathrm{AD})=48 \%$ ), and after wipe cleaning with pure water $(\mathrm{AD}=27.3 \%)$, SDBS surfactant $(\mathrm{AD}=10.8 \%)$ and SDS surfactant $(\mathrm{AD}=7.9 \%)$. (C) Sample SEM image of the surface of SWCNTs deposited on silicon wafers before cleaning $(A D=48 \%)$, and after wipe cleaning with pure water ( $\mathrm{AD}=14.7 \%)$, SDBS surfactant $(\mathrm{AD}=3.2 \%)$ and SDS surfactant $(\mathrm{AD}=2.1 \%)$. (D) Sample SEM images of the surface of SWCNHs deposited on silicon wafers before cleaning $(\mathrm{AD}=36 \%)$, and after wipe cleaning with pure water $(\mathrm{AD}=12.5 \%)$, SDBS surfactant $(\mathrm{AD}=1.5 \%)$ and $\operatorname{SDS}$ surfactant $(\mathrm{AD}=1.2 \%)$. 
(A)

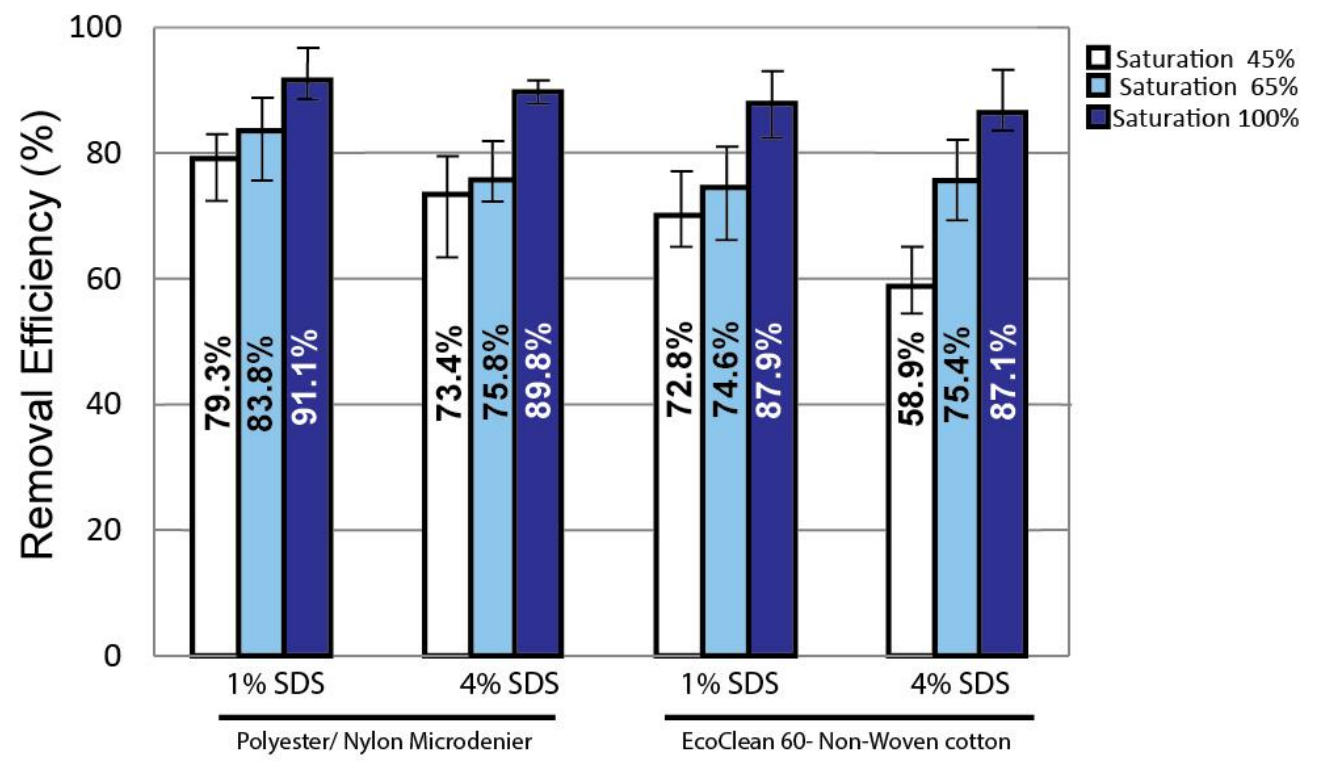

(B)

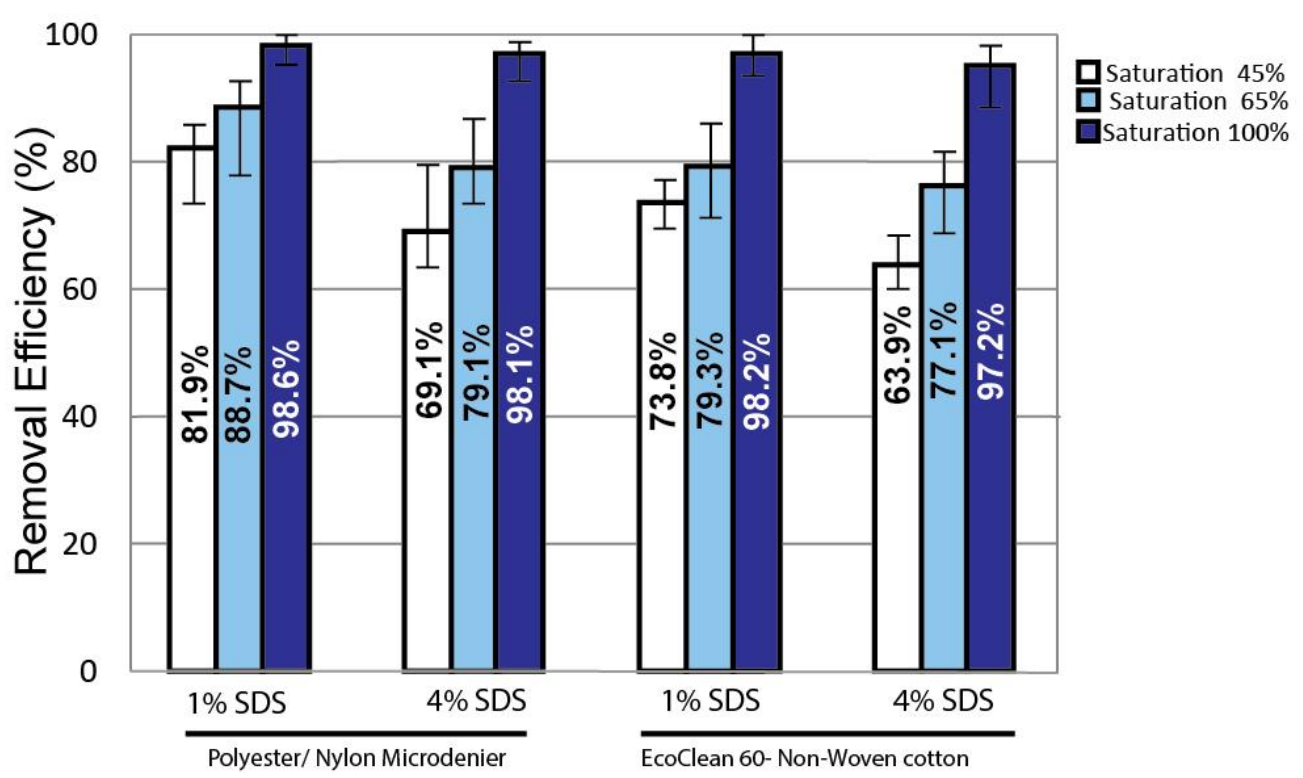

Fig. 4. (A) SWCNHs removal efficiency by using wet wipes, Polyester/ Nylon Microdenier with $4 \%$ and $1 \%$ SDS concentration and 45\%, 65\% and 100\% saturation and EcoClean 60 - non-woven (B) MWCNT removal efficiency by using wet wipes, Polyester/ Nylon Microdenier with $4 \%$ and $1 \%$ SDS concentration and $45 \%, 65 \%$ and $100 \%$ saturation and EcoClean 60 - non-woven cotton 


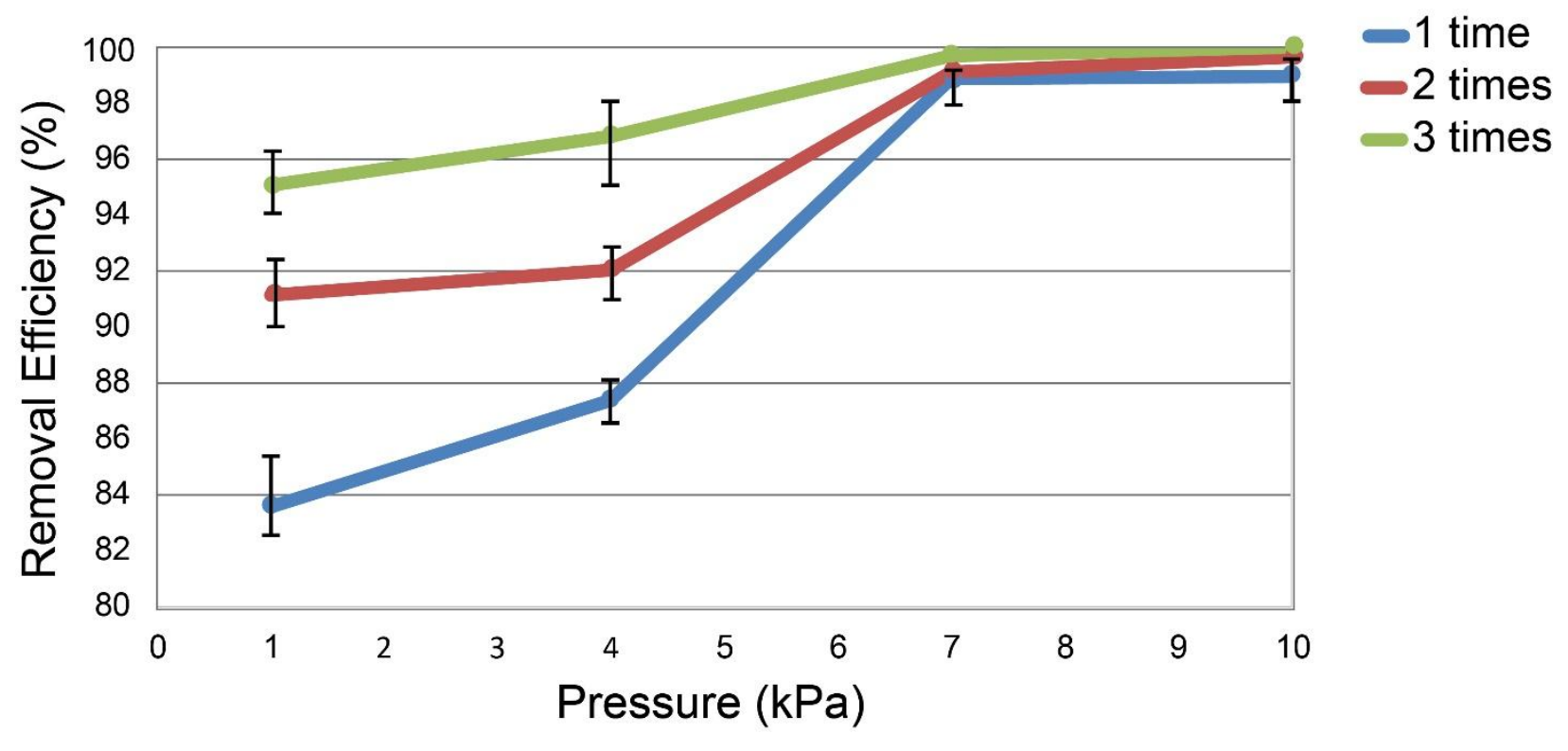

Fig. 5. Removal efficiency of the deposited SWCNHs on the silicon wafer after a single cleaning (blue line), 2 times cleaning (red line), and 3 times cleaning (green line). 


\section{References}

1. A. Jorio, G. Dresselhaus and M. S. Dresselhaus, Carbon nanotubes: advanced topics in the synthesis, structure, properties and applications, Springer Science \& Business Media, 2007.

2. M. Das, N. Saxena and P. D. Dwivedi, "Emerging trends of nanoparticles application in food technology: Safety paradigms," Nanotoxicology, vol. 3, no. 1, pp. 10-18, 2009.

3. K. Balasubramanian and M. Burghard, "Chemically functionalized carbon nanotubes," Small, vol. 1, no. 2, pp. 180-192, 2005.

4. M. S. Dresselhaus and P. Avouris, "Introduction to carbon materials research," in Carbon nanotubes, Ed., pp. 1-9, Springer, 2001.

5. R. S. Ruoff, D. Qian and W. K. Liu, "Mechanical properties of carbon nanotubes: theoretical predictions and experimental measurements," Comptes Rendus Physique, vol. 4, no. 9, pp. 993-1008, 2003.

6. J.-P. Salvetat, J.-M. Bonard, N. Thomson, A. Kulik, L. Forro, W. Benoit and L. Zuppiroli, "Mechanical properties of carbon nanotubes," Applied Physics A, vol. 69, no. 3, pp. 255-260, 1999.

7. C. H. Hsu, C.-F. Chen, C.-C. Chen and S.-Y. Chan, "Density-controlled carbon nanotubes," Diamond and related materials, vol. 14, no. 3, pp. 739-743, 2005.

8. M. J. Treacy, T. Ebbesen and J. Gibson, "Exceptionally high Young's modulus observed for individual carbon nanotubes," 1996.

9. S. Iijima, M. Yudasaka, R. Yamada, S. Bandow, K. Suenaga, F. Kokai and K. Takahashi, "Nanoaggregates of single-walled graphitic carbon nano-horns," Chemical Physics Letters, vol. 309, no. 3, pp. 165-170, 1999.

10. J. A. Nisha, M. Yudasaka, S. Bandow, F. Kokai, K. Takahashi and S. Iijima, "Adsorption and catalytic properties of single-wall carbon nanohorns," Chemical Physics Letters, vol. 328, no. 4, pp. 381-386, 2000.

11. K. Suzuki, M. Yamaguchi, M. Kumagai and S. Yanagida, "Application of Carbon Nanotubes to Counter Electrodes of Dye-sensitized Solar Cells," Chemistry Letters, vol. 32, no. 1, pp. 28-29, 2003.

12. C.-M. Yang, Y.-J. Kim, M. Endo, H. Kanoh, M. Yudasaka, S. Iijima and K. Kaneko, "Nanowindowregulated specific capacitance of supercapacitor electrodes of single-wall carbon nanohorns," Journal of the American Chemical Society, vol. 129, no. 1, pp. 20-21, 2007.

13. M. G. Harisinghani, J. Barentsz, P. F. Hahn, W. M. Deserno, S. Tabatabaei, C. H. van de Kaa, J. de la Rosette and R. Weissleder, "Noninvasive detection of clinically occult lymph-node metastases in prostate cancer," New England Journal of Medicine, vol. 348, no. 25, pp. 2491-2499, 2003.

14. A. Dowling, R. Clift, N. Grobert, D. Hutton, R. Oliver, O. O’Neill, J. Pethica, N. Pidgeon, J. Porritt and J. Ryan, "Nanoscience and nanotechnologies: opportunities and uncertainties," London: The Royal Society \& The Royal Academy of Engineering Report, pp. 61-64, 2004.

15. J. Miyawaki, M. Yudasaka, T. Azami, Y. Kubo and S. Iijima, "Toxicity of single-walled carbon nanohorns," Acs Nano, vol. 2, no. 2, pp. 213-226, 2008.

16. R. M. Lynch, B. H. Voy, D. F. Glass, S. M. Mahurin, B. Zhao, H. Hu, A. M. Saxton, R. L. Donnell and M.-D. Cheng, "Assessing the pulmonary toxicity of single-walled carbon nanohorns," Nanotoxicology, vol. 1, no. 2, pp. 157-166, 2007.

17. K. Kostarelos, "The long and short of carbon nanotube toxicity," Nature biotechnology, vol. 26, no. 7 , pp. 774-776, 2008.

18. S.-T. Yang, X. Wang, G. Jia, Y. Gu, T. Wang, H. Nie, C. Ge, H. Wang and Y. Liu, "Long-term accumulation and low toxicity of single-walled carbon nanotubes in intravenously exposed mice," Toxicology letters, vol. 181, no. 3, pp. 182-189, 2008.

19. S. I. Entsiklopediya, "Soviet historical encyclopedia," Moscow, Izdat." Sov. Ents, vol. 7, 1961.

20. B. Bhushan, Tribology Issues and Opportunities in MEMS: Proceedings of the Nsf/Afosr/Asme Workshop on Tribology Issues and Opportunities in Mems Held in Columbus, Ohio, USA, 9-11 November 1997, Springer Science \& Business Media, 1998. 
21. S. Krishnan, A. Busnaina, D. Rimai and L. Demejo, "The adhesion-induced deformation and the removal of submicrometer particles," Journal of adhesion science and technology, vol. 8, no. 11, pp. 1357-1370, 1994.

22. M. Beck, G. Mandi and S. Keki, "Solubility and Molecular Structure State of C60 Organic Solvents," in 187th Electrochemical Society Meeting, Reno, NV, Ed., 1995.

23. P. Su, B. Haghpanah, W. W. Doerr, Z. Karimi, S. Hassan, L. Gritzo, A. A. Busnaina and A. Vaziri, "Decontamination of surfaces exposed to carbon-based nanotubes and nanomaterials," Journal of Nanomaterials, vol. 2014, pp. 1, 2014.

24. Z. Karimi, P. Su, S. Hassan, B. Haghpanah, W. Doerr and A. Vaziri, "Removal of multiwalled carbon nanotube contaminants from surfaces with microscale topological features," Environmental Progress \& Sustainable Energy, vol. 35, no. 1, pp. 161-168, 2016.

25. M. J. O'Connell, S. M. Bachilo, C. B. Huffman, V. C. Moore, M. S. Strano, E. H. Haroz, K. L. Rialon, P. J. Boul, W. H. Noon and C. Kittrell, "Band gap fluorescence from individual single-walled carbon nanotubes," Science, vol. 297, no. 5581, pp. 593-596, 2002.

26. R. Rastogi, R. Kaushal, S. Tripathi, A. L. Sharma, I. Kaur and L. M. Bharadwaj, "Comparative study of carbon nanotube dispersion using surfactants," Journal of colloid and interface science, vol. 328, no. 2, pp. 421-428, 2008.

27. M. J. Rosen and J. T. Kunjappu, Surfactants and interfacial phenomena, John Wiley \& Sons, 2012.

28. P. Somasundaran, T. t. Healy and D. Fuerstenau, "The aggregation of colloidal alumina dispersions by adsorbed surfactant ions," Journal of colloid and interface science, vol. 22, no. 6, pp. 599-605, 1966.

29. Z. Sun, V. Nicolosi, D. Rickard, S. D. Bergin, D. Aherne and J. N. Coleman, "Quantitative evaluation of surfactant-stabilized single-walled carbon nanotubes: dispersion quality and its correlation with zeta potential," The Journal of Physical Chemistry C, vol. 112, no. 29, pp. 10692-10699, 2008. 